\title{
Is comorbid depression related to weight gain during treatment of anorexia nervosa?
}

\author{
Adrian Meule ${ }^{1,2}$, Sandra Schlegl'1, \& Ulrich Voderholzer ${ }^{1,2,3}$ \\ 'Department of Psychiatry and Psychotherapy, University Hospital, LMU Munich, Munich, Germany \\ Schoen Clinic Roseneck, Prien am Chiemsee, Germany \\ ${ }^{3}$ Department of Psychiatry and Psychotherapy, University Hospital of Freiburg, Freiburg, Germany
}

In a recent review article, Eskild-Jensen et al. (2020) aimed to examine "comorbid depression as a negative predictor of weight gain during treatment of anorexia nervosa" (cf. title, p. 605) or more generally "the influence of psychiatric comorbidity on weight gain during treatment of anorexia nervosa" (cf. abstract, p. 605). Only few studies (15) were included in the review and the authors conclude that studies were heterogeneous in design, purpose, and outcome. This finding alone may lead readers to conclude that there is no consistent evidence that comorbid depression may negatively affect weight gain in anorexia nervosa. However, the authors highlight prominently that "depression had a negative influence on weight gain in four studies" (final sentence of the abstract, p. 605) and that "four well-designed studies found that depression is a negative factor for weight gain" (last bullet point in the highlights, p. 606), thus implying that although studies and findings were heterogeneous, there is at least some evidence that comorbid depression negatively affects weight gain during treatment of anorexia nervosa. In addition, the authors state that "overall, not taking level of evidence and bias into account, five studies found that depression was a negative predictor of weight gain (Berona et al., 2018; Keski-Rahkonen et al., 2014; Lock et al., 2006; Schlegl et al., 2014; Schlegl et al., 2016), whilst one study found that moderate depression (Schlegl et al., 2014) was a positive predictor" (p. 614) and that "overall, depression was the comorbid disorder that most clearly had a negative influence on weight gain, both in the short-term and long-term studies" (p. 614). In this commentary, we argue that these conclusions are unwarranted.
In the study by Berona et al. (2018), patients were categorized in three groups based on body weight data during treatment: slow, moderate, and rapid weight gain. The slow weight gain group had higher rates of comorbid mood disorders than the other two groups. However, the slow weight gain group also had the highest body mass index at admission. Thus, it is unclear whether comorbid mood disorders may actually affect weight change or whether the slow weight gain may simply be explained by the fact that these patients already started with a higher body weight at admission. Nevertheless, the statement that depression related to smaller weight gain in this study is still technically correct.

The study by Keski-Rahkonen et al. (2014) was not a treatment study but a naturalistic observational study with data from the general population. One of the findings was that women who were recovered from anorexia nervosa were less likely to have depressive symptoms prior to eating disorder onset than women who were not recovered. Recovery was defined as restoration of weight, menstruation, and the absence of bingeing and purging for at least one year prior to assessment. Thus, this study did not investigate treatment effects and-as recovery was defined by several criteria-did not investigate weight gain.

The study by Lock et al. (2006) investigated predictors of dropout and remission during treatment of anorexia nervosa. Those with a psychiatric comorbid disorder were less likely to remit. However, remission was defined as being at greater than $95 \%$ ideal body weight and having a total Eating Disorder Examination score within two standard deviations of community norms at the end of treatment. Thus-as re-

Copyright @ 2020 Adrian Meule. This is an open-access article distributed under the terms of the Creative Commons Attribution License (CC BY). The use, distribution or reproduction in other forums is permitted, provided the original author(s) and the copyright owner(s) are credited and that the original publication in this journal is cited, in accordance with accepted academic practice. No use, distribution or reproduction is permitted which does not comply with these terms.

Adrian Meule (ه) ameule@med.Imu.de

Received: 23 November 2020; Accepted: 30 November 2020; Published online: 21 December 2020. doi: 10.32044/ijedo.2020.07 
mission was not only defined by achieving a certain weightthis study's findings do not allow to conclude that comorbid depression related to weight gain during treatment.

The findings by Schlegl et al. (2016) are cited such that depression negatively predicted clinically significant change during inpatient treatment of anorexia nervosa (see Table 1 on p. 609). However, this is incorrect because the analyses on predictors of clinically significant change referred to scores on the Eating Disorder Inventory-2 and not to weight change. In the article by Schlegl et al. (2016), variables that related to weight change are actually reported but only comorbid social phobia-and not depression -related to change in body mass index.

Similarly, the findings by Schlegl et al. (2014) are cited such that moderate depression was a positive predictor and severe depression was a negative predictor of clinically significant change. However, this is again incorrect because the analyses on predictors of clinically significant change referred to scores on the Eating Disorder Inventory-2 and not to weight change. This misreporting is particularly crucial as the authors prominently highlight this study in the abstract, highlights, and conclusion as the only study that identified moderate depression as a positive predictor of weight gain (pp. 605, 606, 618).

We conclude that one of the main findings presented by Eskild-Jensen et al. (2020) is incorrect. Specifically, only one study (Berona et al., 2018) found an association between comorbid depressive disorders and weight gain during treatment of anorexia nervosa. The other studies that the authors cite as showing an effect of depression on weight gain during treatment of anorexia nervosa did actually not investigate this or did not find such an effect. Thus, we would argue that the main conclusion of this review should be that the large majority of studies did not find or report that depression relates to weight change during treatment in patients with anorexia nervosa.

\section{References}

Berona, J., Richmond, R., \& Rienecke, R. D. (2018). Heterogeneous weight restoration trajectories during partial hospitalization treatment for anorexia nervosa. International Journal of Eating Disorders, 51, 914-920. https://doi.org/10.1002/eat.22922

Eskild-Jensen, M., Støving, R. K., Flindt, C. F., \& Sjogren, M. (2020). Comorbid depression as a negative predictor of weight gain during treatment of anorexia nervosa: A systematic scoping review. European Eating Disorders Review, 28, 605-619. https://doi.org/10.1002/erv.2787

Keski-Rahkonen, A., Raevuori, A., Bulik, C. M., Hoek, H. W., Rissanen, A., \& Kaprio, J. (2014). Factors associated with recovery from anorexia nervosa: A populationbased study. International Journal of Eating Disorders, 47, 117-123. https://doi.org/10.1002/eat.22168

Lock, J., Couturier, J., Bryson, S., \& Agras, S. (2006). Predictors of dropout and remission in family therapy for adolescent anorexia nervosa in a randomized clinical trial. International Journal of Eating Disorders, 39, 639-647. https://doi.org/10.1002/eat.20328

Schlegl, S., Diedrich, A., Neumayr, C., Fumi, M., Naab, S., \& Voderholzer, U. (2016). Inpatient treatment for adolescents with anorexia nervosa: clinical significance and predictors of treatment outcome. European Eating Disorders Review, 24, 214-222. https://doi.org/10.1002/ erv.2416

Schlegl, S., Quadflieg, N., Löwe, B., Cuntz, U., \& Voderholzer, U. (2014). Specialized inpatient treatment of adult anorexia nervosa: effectiveness and clinical significance of changes. BMC Psychiatry, 14(258), 1-12. https://doi.org/10.1186/s12888-014-0258-z 